21st Particles and Nuclei International Conference (PANIC 2017)

International Journal of Modern Physics: Conference Series

Vol. 46 (2018) 1860043 (8 pages)

(C) The Author(s)

DOI: $10.1142 / \mathrm{S} 2010194518600431$

\title{
Limits on Heavy Neutrinos at NA48/2 and NA62
}

\author{
Lubos Bician*, $†$ \\ Faculty of Mathematics, Physics and Informatics, Comenius University \\ Mlynska dolina F1, Bratislava, 842 48, Slovakia \\ lubos.bician@fmph.uniba.sk
}

Published 3 May 2018

Large samples of charged kaon decays have been collected by the NA48/2 (2003-2004), the NA62-R $\mathrm{K}_{\mathrm{K}}(2007)$ and the NA62 (2015) experiments at CERN SPS. This proceedings

\begin{abstract}
* Speaker.
${ }^{\dagger}$ for the NA62 Collaboration: R. Aliberti, F. Ambrosino, R. Ammendola, B. Angelucci, A. Antonelli, G. Anzivino, R. Arcidiacono, M. Barbanera, A. Biagioni, L. Bician, C. Biino, A. Bizzeti, T. Blazek, B. Bloch-Devaux, V. Bonaiuto, M. Boretto, M. Bragadireanu, D. Britton, F. Brizioli, M.B. Brunetti, D. Bryman, F. Bucci, T. Capussela, A. Ceccucci, P. Cenci, V. Cerny, C. Cerri, B. Checcucci, A. Conovaloff, P. Cooper, E. Cortina Gil, M. Corvino, F. Costantini, A. Cotta Ramusino, D. Coward, G. D'Agostini, J. Dainton, P. Dalpiaz, H. Danielsson, N. De Simone, D. Di Filippo, L. Di Lella, N. Doble, B. Dobrich, F. Duval, V. Duk, J. Engelfried, T. Enik, N. Estrada-Tristan, V. Falaleev, R. Fantechi, V. Fascianelli, L. Federici, S. Fedotov, A. Filippi, M. Fiorini, J. Fry, J. Fu, A. Fucci, L. Fulton, E. Gamberini, L. Gatignon, G. Georgiev, S. Ghinescu, A. Gianoli, M. Giorgi, S. Giudici, F. Gonnella, E. Goudzovski, C. Graham, R. Guida, E. Gushchin, F. Hahn, H. Heath, T. Husek, O. Hutanu, D. Hutchcroft, L. Iacobuzio, E. Iacopini, E. Imbergamo, B. Jenninger, K. Kampf, V. Kekelidze, S. Kholodenko, G. Khoriauli, A. Khotyantsev, A. Kleimenova, A. Korotkova, M. Koval, V. Kozhuharov, Z. Kucerova, Y. Kudenko, J. Kunze, V. Kurochka, V.Kurshetsov, G. Lanfranchi, G. Lamanna, G. Latino, P. Laycock, C. Lazzeroni, M. Lenti, G. Lehmann Miotto, E. Leonardi, P. Lichard, L. Litov, R. Lollini, D. Lomidze, A. Lonardo, P. Lubrano, M. Lupi, N. Lurkin, D. Madigozhin, I. Mannelli, G. Mannocchi, A. Mapelli, F. Marchetto, R. Marchevski, S. Martellotti, P. Massarotti, K. Massri, E. Maurice, M. Medvedeva, A. Mefodev, E. Menichetti, E. Migliore, E. Minucci, M. Mirra, M. Misheva, N. Molokanova, M. Moulson, S. Movchan, M. Napolitano, I. Neri, F. Newson, A. Norton, M. Noy, T. Numao, V. Obraztsov, A. Ostankov, S. Padolski, R. Page, V. Palladino, C. Parkinson, E. Pedreschi, M. Pepe, M. Perrin-Terrin, L. Peruzzo, P. Petrov, F. Petrucci, R. Piandani, M. Piccini, J. Pinzino, I. Polenkevich, L. Pontisso, Yu. Potrebenikov, D. Protopopescu, M. Raggi, A. Romano, P. Rubin, G. Ruggiero, V. Ryjov, A. Salamon, C. Santoni, G. Saracino, F. Sargeni, V. Semenov, A. Sergi, A. Shaikhiev, S. Shkarovskiy, D. Soldi, V. Sougonyaev, M. Sozzi, T. Spadaro, F. Spinella, A. Sturgess, J. Swallow, S. Trilov, P. Valente, B. Velghe, S. Venditti, P. Vicini, R. Volpe, M. Vormstein, H. Wahl, R. Wanke, B. Wrona, O. Yushchenko, M. Zamkovsky, A. Zinchenko.
\end{abstract}

This is an Open Access article published by World Scientific Publishing Company. It is distributed under the terms of the Creative Commons Attribution 4.0 (CC-BY) License. Further distribution of this work is permitted, provided the original work is properly cited. 


\section{Bician}

summarizes upper limits on heavy neutrino production measured by these experiments. The results from the 2015 data analysis at the NA62 experiment are preliminary.

Keywords: NA62; NA48/2; heavy neutrinos.

\section{Introduction}

The Standard Model (SM) of particle physics ${ }^{1-3}$ does not describe the observed phenomenon of neutrino oscillations ${ }^{4,5}$ because neutrinos are strictly massless in the SM and therefore do not oscillate. One of the extensions of the SM, capable of accommodating non-zero SM neutrino masses, is the Neutrino Minimal Standard $\operatorname{Model}^{6}(\nu \mathrm{MSM})$, where three sterile massive right-handed neutrinos are added to the SM. The lightest $\left(\mathcal{O}\left(1 \mathrm{keV} / c^{2}\right)\right)$ of the sterile neutrinos serves as a dark matter candidate, while the other two $\left(\mathcal{O}\left(1 \mathrm{GeV} / c^{2}\right)\right)$ generate SM neutrino masses via the see-saw mechanism. ${ }^{7}$ The model introduces additional CPV phases which could explain the observed baryon asymmetry of the Universe.

Due to the mixing between Heavy Neutral Leptons (HNLs) and SM neutrinos, HNLs could be produced in meson decays such as $K^{+} \rightarrow l^{+} N(l=e, \mu)$. The branching fraction of the production processes $K^{+} \rightarrow l^{+} N$ can be expressed in terms of the branching fraction of the SM $K^{+} \rightarrow l^{+} \nu_{l}\left(K_{l 2}\right)$ decays using kinematic factor $\rho_{l}\left(m_{N}\right)$ accounting for the helicity suppression and phase space dependence on the HNL mass $m_{N}$, and the mixing parameter $\left|U_{l 4}\right|^{2}$ :

$$
\mathcal{B}\left(K^{+} \rightarrow l^{+} N\right)=\mathcal{B}\left(K^{+} \rightarrow l^{+} \nu_{l}\right) \cdot \rho_{l}\left(m_{N}\right) \cdot\left|U_{l 4}\right|^{2} .
$$

This proceedings describes three separate analyses performed at the NA48/2 experiment $^{8}$ and, its successor, the NA62 experiment at CERN SPS, aiming to observe HNL production (and decay). The search for HNLs at the NA48/2 (see Ref. 8) was carried out on the three-track data collected in 2003 and 2004, searching for the production and subsequent decay of HNLs. The analysis of the NA62-R data aimed to observe HNL production in $K^{+} \rightarrow \mu^{+} N$ decays in 2007 data. ${ }^{9}$ This proceedings also presents preliminary results from the HNL production search in $K^{+} \rightarrow e^{+} N$ decays in the data collected in 2015 at the NA62 experiment.

\section{Heavy Neutrino Searches at NA48/2}

The NA48/2 was a charged kaon experiment at CERN SPS with the main goal of studying direct CP violation in $K^{ \pm} \rightarrow \pi^{ \pm} \pi^{+} \pi^{-}$and $K^{ \pm} \rightarrow \pi^{ \pm} \pi^{0} \pi^{0}$ decays. The large statistics of kaon decays collected in years 2003 and 2004 allowed for the HNL search in decays with three charged tracks. A HNL produced in $K^{ \pm} \rightarrow \mu^{ \pm} N$ and decaying promptly into an oppositely charged particle pair $\pi^{\mp} \mu^{ \pm}$can mediate Lepton Number Violating (LNV) decay $K^{ \pm} \rightarrow \pi^{\mp} \mu^{ \pm} \mu^{ \pm}\left(K_{\pi \mu \mu}^{\mathrm{LNV}}\right)$. Likewise, HNL produced in the same decay but decaying into $\pi^{ \pm} \mu^{\mp}$ pair would produce a narrow peak in the invariant mass spectrum $M_{\pi \mu}$ of the Lepton Number Conserving (LNC) decay $K^{ \pm} \rightarrow \pi^{ \pm} \mu^{+} \mu^{-}\left(K_{\pi \mu \mu}^{\mathrm{LNC}}\right)$. LNV and LNC decay selections have been developed 
and subsequently the invariant mass spectra $M_{\pi \mu}$ have been scanned for a HNL signature.

\subsection{Experimental Setup and Data Taking Conditions}

Simultaneous and collinear $K^{+}$and $K^{-}$beams with the momentum of $(60 \pm$ 3.7) $\mathrm{GeV} / c$ were produced from the primary $400 \mathrm{GeV} / c$ SPS protons and subsequently steered into a $114 \mathrm{~m}$ long evacuated cylindrical decay region. The downstream part of the vacuum tank was sealed from a helium tank at atmospheric pressure, in which a magnetic spectrometer consisting of four drift chambers (DCHs) and a dipole magnet were housed. The spectrometer was followed by a hodoscope (HOD) used in the online trigger system and as the offline time reference for the charged particles. A Liquid Krypton electromagnetic calorimeter (LKr) was placed downstream of the HOD detector. The LKr was followed by a muon detector (MUV) placed behind a $80 \mathrm{~cm}$ thick iron wall. A detailed description of the NA48/2 beam line and detector layout can be found in Refs. 10 and 11.

\subsection{Event Selection}

The event selection is based on the reconstruction of a three-track vertex. Due to the limited resolution on the longitudinal position of the vertex $\left(\sigma_{\mathrm{vtx}}=0.5 \mathrm{~m}\right)$, it is impossible to distinguish a genuine three-track decay from the one mediated by a short-lived $(\tau \lesssim 10 \mathrm{ps})$ HNL that produces a pair of charged particles. The three decays of interest are: $K_{\pi \mu \mu}^{\mathrm{LNC}}, K_{\pi \mu \mu}^{\mathrm{LNV}}$ and the abundant decay $K^{ \pm} \rightarrow \pi^{ \pm} \pi^{+} \pi^{-}$ $\left(K_{3 \pi}\right)$ used for normalization.

The $K_{3 \pi}$ and $K_{\pi \mu \mu}$ event selections share a common part: at least one threetrack vertex with the total charge $Q= \pm 1$ and the total (transverse) momentum consistent with the nominal beam momentum has to be reconstructed inside the fiducial volume. If there are multiple vertices satisfying these conditions, the one with the lowest fit $\chi^{2}$ is chosen.

Muon and pion particle identification in the $K_{\pi \mu \mu}^{\mathrm{LNC}}\left(K_{\pi \mu \mu}^{\mathrm{LNV}}\right)$ event selection is done using hits in the MUV and the ratio of the energy $E$ deposited in the LKr and the momentum $p$ measured by the DCH. The invariant mass of the three tracks $M_{\pi \mu \mu}$ has to be consistent with the is the nominal kaon mass $M_{K} \cdot{ }^{12}$

Independently of the $K_{\pi \mu \mu}$-related requirements, the $K_{3 \pi}$ sample selection contains the following criteria: the pion identification described above is applied only to the even-signed (odd-signed) pion to ensure symmetry between the signal and normalization modes, and the invariant mass $M_{3 \pi}$ of the three tracks under the $3 \pi^{ \pm}$ hypothesis must be consistent with $M_{K}$.

\subsection{Selected Samples and Heavy Neutrino Signal Searches}

The invariant mass spectra for data and MC events passing the $K_{\pi \mu \mu}^{\mathrm{LNC}}$ and $K_{\pi \mu \mu}^{\mathrm{LNV}}$ selections are shown in Fig. 1. There is one event present inside the signal region of 

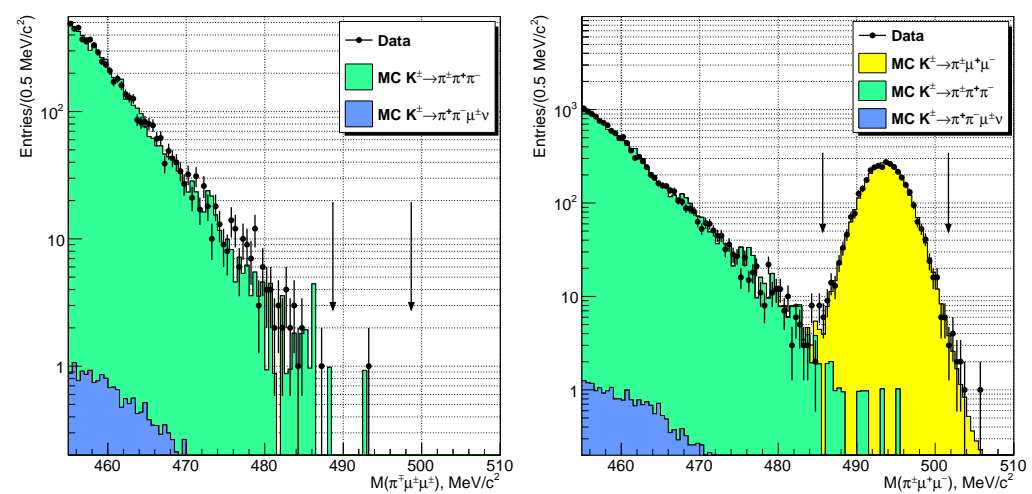

Fig. 1. Invariant mass spectra of the $K_{\pi \mu \mu}^{\mathrm{LNC}}$ and $K_{\pi \mu \mu}^{\mathrm{LNV}}$ data and MC events. Arrows indicate signal regions.

the $K_{\pi \mu \mu}^{\mathrm{LNV}}$ sample, while the $K_{\pi \mu \mu}^{\mathrm{LNC}}$ sample contains 3489 candidates in the signal region. The number of expected background events in the $K_{\pi \mu \mu}^{\mathrm{LNV}}$ sample (mostly from $K_{3 \pi}$ decays with two subsequent $\pi \rightarrow \mu \nu$ decays) is $N_{\mathrm{bkg}}=1.163 \pm 0.875$. The $K_{\pi \mu \mu}^{\mathrm{LNC}}$ sample contamination is $(0.36 \pm 0.10) \%$. The number of kaon decays in the fiducial volume, calculated using the $K_{3 \pi}$ normalization channel, is $N_{K}=$ $(1.64 \pm 0.01) \times 10^{11}$.

\subsection{Limits on HNL production and decay}

A search for resonances assuming different HNL masses is performed in the invariant mass spectra $M_{i j}$ of $\pi^{ \pm} \mu^{\mp}\left(\pi^{\mp} \mu^{ \pm}\right)$pairs in the $K_{\pi \mu \mu}^{\mathrm{LNC}}\left(K_{\pi \mu \mu}^{\mathrm{LNV}}\right)$ samples. The mass step is chosen to be $\sigma\left(M_{i j}\right) / 2$ and the signal window is defined by $\left|M_{i j}-M_{N}\right|<2 \sigma\left(M_{i j}\right)$, where $M_{N}$ is the assumed HNL mass. Since no statistically significant signal is found for any of the tested mass hypotheses, an extension of the Rolke-López method ${ }^{13}$ is used to estimate the $90 \%$ confidence level (CL) upper limits (ULs) on the number of HNL signal events $N_{\text {sig. }}$. These ULs are subsequently converted into the ULs on branching fractions using the HNL mass dependent acceptance evaluated from $\mathrm{MC}$ and the total number of kaon decays $N_{K}$ defined above. The obtained ULs on branching fractions for various HNL lifetime assumptions are shown in Fig. 2. Finally, the ULs on the branching fractions are translated into ULs on the mixing parameter $\left|U_{l 4}\right|^{2}$, shown in Fig. 3 .

\section{Heavy Neutrino Searches at NA62-R $\mathbf{K}_{\mathrm{K}}$ and NA62}

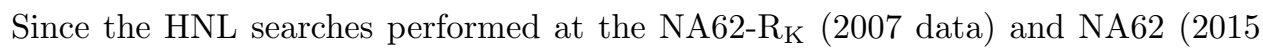
data) used similar approaches, they will both be described side-by-side in this section. 

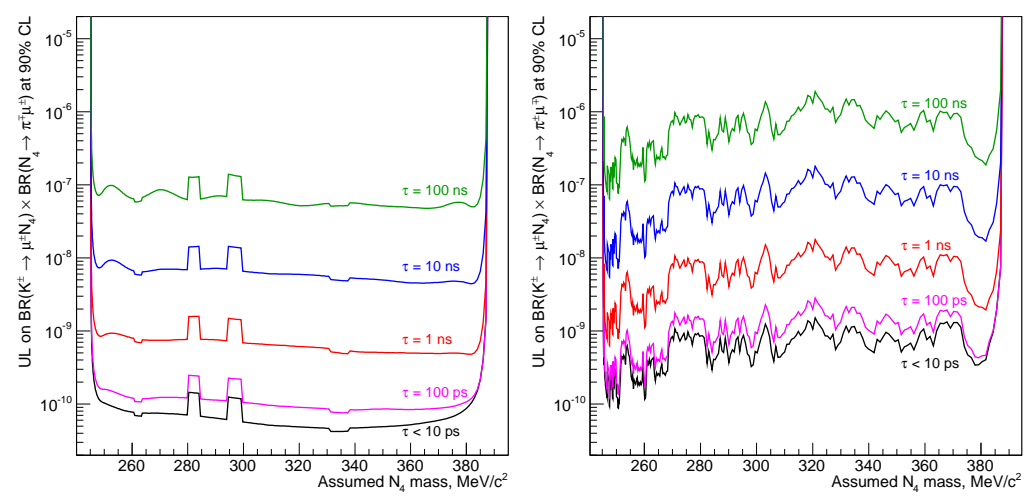

Fig. 2. ULs on the branching fractions products $\mathcal{B}\left(K^{ \pm} \rightarrow \mu^{ \pm} N\right) \times \mathcal{B}\left(N \rightarrow \pi^{\mp} \mu^{ \pm}\right)$(left, LNV) and $\mathcal{B}\left(K^{ \pm} \rightarrow \mu^{ \pm} N\right) \times \mathcal{B}\left(N \rightarrow \pi^{ \pm} \mu^{\mp}\right)$ (right, LNC) for several tested HNL lifetimes.
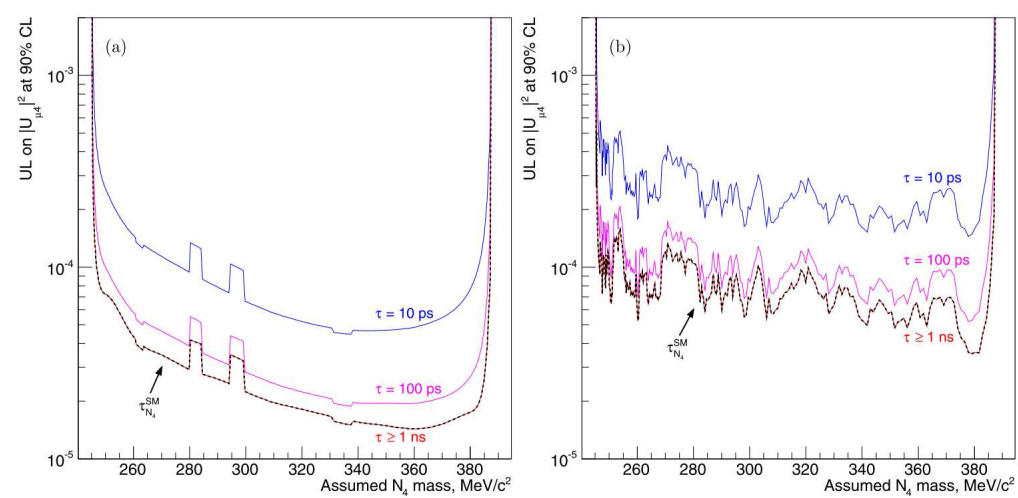

Fig. 3. ULs on the mixing parameter $\left|U_{l 4}\right|^{2}$ for the LNV sample (left) and the LNC sample (right) for several tested HNL lifetimes.

\subsection{Experimental Setup and Data Taking Conditions}

The NA62-R $\mathrm{R}_{\mathrm{K}}$ detector setup was similar to the one used in NA48/2, however several improvements and modifications were made. The NA62- $\mathrm{R}_{\mathrm{K}}$ nominal beam momentum was increased to $(74.0 \pm 1.4) \mathrm{GeV} / c$ and the possibility to switch between $K^{+}, K^{-}$and simultaneous $K^{+}$and $K^{-}$beams was introduced. DCH resolution and other detector parameters were improved as well. HNL search on 2007 data was done only on the $K^{+}$sample.

The NA62 is the most recent kaon experiment at CERN SPS with the aim of a precise measurement of $\mathcal{B}\left(K^{+} \rightarrow \pi^{+} \nu \bar{\nu}\right)$. Compared to its predecessors, it has a significantly improved detector design, shown in Fig. 4. The main difference in the design is the KTAG system used for positive identification of $K^{+}$in the beam $\left(75.0 \pm 1.0 \mathrm{GeV} / c, \sim 6 \% K^{+}\right)$with respect to other beam particles (mainly protons and pions) and the Gigatracker (GTK, not available in 2015) detector designed for 


\section{Bician}

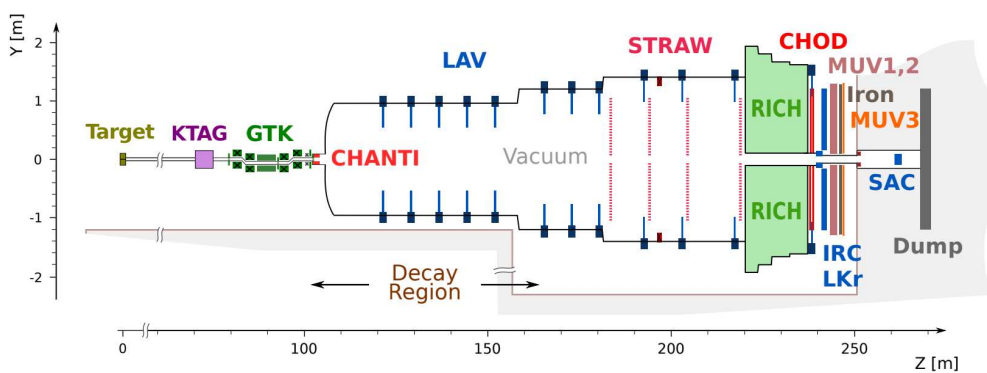

Fig. 4. The NA62 detector layout.
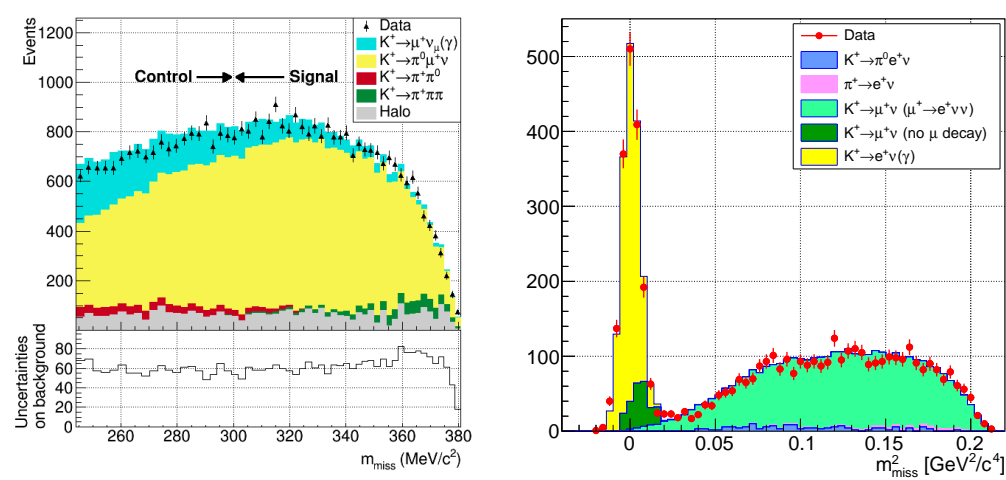

Fig. 5. Missing mass distribution of the selected $K_{\mu 2}$ events and MC background estimates together with their errors for 2007 analysis (left) and missing mass squared spectrum of the selected $K_{e 2}$ candidates from 2015 analysis (right).

precise beam momentum measurement. Other subdetectors involve magnetic spectrometer STRAW consisting of four chambers of drift tubes operating in vacuum, a system of photon vetoes LAV, LKr, IRC and SAC, a RICH detector and a muon veto system MUV. For detailed description of the NA62 detector, see Ref. 14.

\subsection{Event Selections}

The search for HNL produced in $K^{+} \rightarrow \mu^{+} N$ decays in 2007 data is performed on the sample obtained at $\sim 10$ times lower beam intensity compared to the NA48/2 in order to suppress accidental background and ensure high minimum bias trigger efficiency. The analysis requires one well reconstructed positive track identified as muon using MUV hits.

The 2015 data analysis is performed on the minimum bias sample recorded during five days at $\sim 1 \%$ nominal NA62 beam intensity and focuses on the $K^{+} \rightarrow$ $e^{+} N$ decays with electrons identified using $E / p$ cut and RICH. Several background contributions are suppressed using the LAV, SAC, IRC and CHANTI vetoes and by the positive identification of the decaying kaon in the KTAG. 

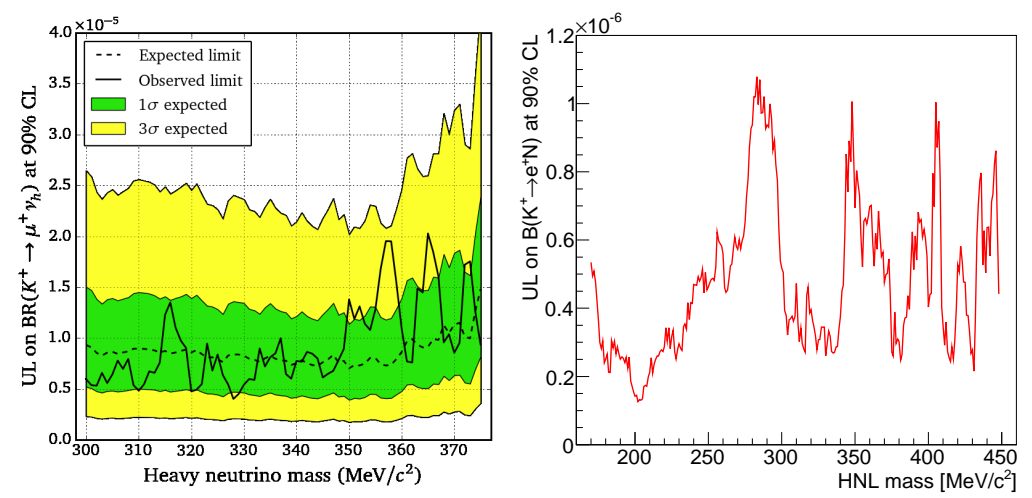

Fig. 6. ULs at $90 \% \mathrm{CL}$ on $\mathcal{B}\left(K^{+} \rightarrow \mu^{+} N\right)$ from 2007 analysis (left) and ULs at $90 \% \mathrm{CL}$ on $\mathcal{B}\left(K^{+} \rightarrow e^{+} N\right)$ from 2015 analysis (right).

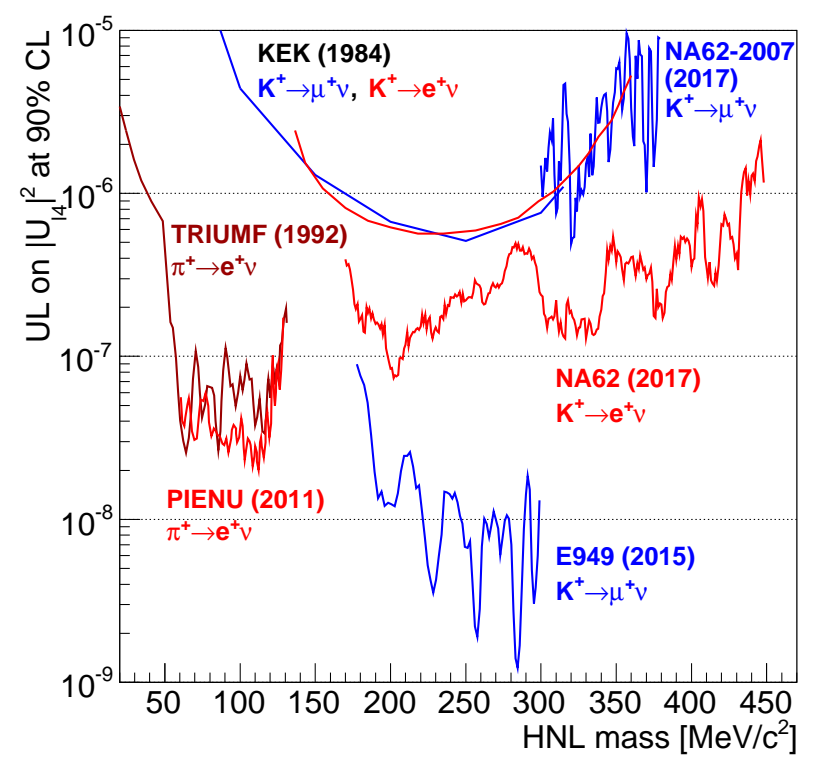

Fig. 7. ULs at $90 \% \mathrm{CL}$ on $\mathcal{B}\left(K^{+} \rightarrow \mu^{+} N\right)$ from 2007 analysis (left) and ULs at $90 \%$ CL on $\mathcal{B}\left(K^{+} \rightarrow e^{+} N\right)$ from 2015 analysis (right). The NA48/2 limits shown in Fig. 3 lie above $10^{-5}$ and so are not shown in the plot.

\subsection{Selected Samples and Heavy Neutrino Signal Searches}

The variable scanned for HNL signal peaks is the missing mass $m_{\text {miss }}=$ $\sqrt{\left(P_{K}-P_{l}\right)^{2}}(l=e, \mu)$, where $P_{K}$ and $P_{l}$ are nominal kaon and lepton fourmomenta respectively. The regions scanned for peaks are $m_{\text {miss }} \in(300,375) \mathrm{MeV} / c^{2}$ (2007 analysis) and $m_{\text {miss }} \in(170,448) \mathrm{MeV} / c^{2}$ (2015 analysis). The step in the mass scan is chosen to be $1 \mathrm{MeV} / c^{2}$ and the half-width of the signal window is proportional to the HNL mass resolution $\sigma_{l}$ evaluated using MC simulations. Missing mass 
(squared) plots obtained from 2007 and 2015 data are shown in Fig. 5. Total number of kaon decays, evaluated using the SM signal regions for $K_{l 2}$ decays and the signal acceptance obtained from MC, are measured to be: $N_{K}^{\mu}=(5.977 \pm 0.015) \times 10^{7}$ (2007 data) and $N_{K}^{e}=(3.01 \pm 0.01) \times 10^{8}(2015$ data $)$.

No statistically significant HNL signal is observed and therefore only ULs at $90 \%$ CL on the number of signal events $N_{\text {sig }}$ are determined for both analyses using the Rolke-López method. ${ }^{13}$ From the ULs on $N_{\text {sig }}$, ULs on the branching fractions of the $K^{+} \rightarrow l^{+} N$ decays are computed (see in Fig. 6) using the HNL mass dependent acceptances and the numbers of kaon decays $N_{K}^{l}$ mentioned above.

The upper limits on the $\mathcal{B}\left(K^{+} \rightarrow l^{+} N\right)$ are used together with Eq. (1) to obtain ULs on the mixing parameters $\left|U_{l 4}\right|^{2}$. The ULs on $\left|U_{\mu 4}\right|^{2}$ and $\left|U_{e 4}\right|^{2}$ obtained from the NA62- $R_{K}$ and NA62 respectively are shown in Fig. 7 and improve the previously established limits.

\section{References}

1. S. Weinberg, Phys. Rev. Lett. 19, 1264 (1967).

2. S.L. Glashow, Nuclear Physics 22, 579 (1961).

3. A. Salam, Conf.Proc. C680519, 367 (1968).

4. Super-Kamiokande Collab. (Y. Fukuda et al.) Phys. Rev. Lett. 81, 1562 (1998).

5. SNO Collab. (Q. R. Ahmad et al.) Phys. Rev. Lett. 87, 071301 (2001).

6. T. Asaka and M. Shaposhnikov, Phys. Lett. B 620, 17 (2005).

7. R.N. Mohapatra and P. B. Pal, World Sci. Lect. Notes Phys. 60, 1 (1998).

8. NA48/2 Collab. (J. R. Batley et al.), Phys. Lett. B 769, 67 (2017).

9. NA62 Collab. (C. Lazzeroni et al.), Phys. Lett. B 772, 712 (2017).

10. NA48/2 Collab. (J. R. Batley et al.), Eur. Phys. J. C 52, 875 (2007).

11. NA48/2 Collab. (V. Fanti et al.), Nucl. Instrum. Methods A 574, 433 (2007).

12. Particle Data Group (C. Patrignani et al.), Chinese Phys. C 40, 100001 (2016).

13. W.A. Rolke and A.M. López, Nucl. Instrum. Methods A 458, 745 (2001).

14. NA62 Collab. (G. Cortina et al.), JINST 12, P05025 (2017). 